\title{
Formal Pipeline Design
}

\author{
Tiberiu Seceleanu and Juha Plosila
}

University of Turku, Dept of Applied Physics, Lab. of Electronics and Information Technology, FIN-20014 Turku, Finland, tel: +358-2-3336954, fax: +358-2-3336950,

\{Tiberiu.Seceleanu, Juha.Plosila\}@utu.fi

\begin{abstract}
The action systems formalism has recently been applied to the area of asynchronous and synchronous VLSI design. In this paper, we study formal aspects of synchronous pipelining. We show how the framework of synchronous action systems can be used to derive a pipelined structure from a non-pipelined specification in a correctness-preserving manner.
\end{abstract}

\section{Introduction}

Pipelining is a very common technique used in digital systems design in order to increase parallelism. It has an important impact on the rate at which data is produced and consumed, a pipelined version allowing a higher speed than the non-pipelined version of the same circuit [7].

Formal methods of concurrent programming become increasingly important in design of complex VLSI systems. In our earlier work, we have shown how the action systems formalism [1] can be applied in design of both self-timed [8] and synchronous circuits $[9,10]$. In this paper, we continue our work on synchronous modeling by focusing on the formal aspects of clocked pipelining. The actions composing a synchronous action system are high-level representations of circuit functionality. One single action may actually map on a very complex hardware implementation, in terms of the time required to perform. Therefore, the device should operate on a large clock period, situation which, often, does not represent a solution. Thus, a further splitting of the execution of the initial action becomes necessary, reflected further in a pipelined realization of the digital device. Here, we present a stepwise procedure by which a non-pipelined synchronous action system specification is transformed into a pipelined form, in a correctness-preserving manner.

\section{Synchronous Action Systems}

The action systems formalism is based on an extended version of Dijkstra's language of guarded commands [5]. A synchronous action system [9] $\mathcal{A}$ has the form sys $\mathcal{A}(g):: \mid\left[\operatorname{var} l\right.$; init $g, l:=g_{0}, l_{0} ;$ do $A_{1} \nabla A_{2}$ od ]|, where $g$ and $l$ are lists of global and local variables initialised to $g_{0}$ and $l_{0}$, respectively, and $A_{1} \nabla A_{2}$ is the synchronous composition of the actions $A_{1}$ and $A_{2}$ which access the variables $g$ and $l$. 
Any of the actions of the synchronous system $\mathcal{A}$ is defined as a non-deterministic assignment, $x:=x^{\prime} . Q$. Here, $Q$ is a boolean relation which determines the value(s) $x^{\prime}$ assigned to the (list of) variable(s) $x$. For example: $Q \widehat{=} x^{\prime}=x+y$, where $y$ is another variable. The synchronous composition operator ' $\nabla$ ' between the actions $A_{i}$ in the above system $\mathcal{A}$ indicates that the write variables of the involved actions are updated simultaneously in an atomic manner. At a lower abstraction level, a synchronous composition consists of a read phase and a write phase which are executed sequentially one after another.

The hardware representation of a synchronous action is represented by a set of registers (D flip-flops), each associated with one of the write variables of the action, and the corresponding combinational logic (specified, under all input situations, by the predicate $Q$ ).

Two synchronous action systems $\mathcal{A}_{1}$ and $\mathcal{A}_{2}$ can be composed using the operator ' $\nabla$ '. The composition $\mathcal{A}_{1} \nabla \mathcal{A}_{2}$ is defined to be a synchronous action system which is composed of the actions of the constituent systems. This system merges the global variables of the components $\mathcal{A}_{i}$ keeping the local variables distinct.

Actions and action systems are intended to be developed in a stepwise manner within the refinement calculus [2]. A correct transformation of a given action $A$ into the action $A^{\prime}$ is denoted as $A \leq A^{\prime}$. Refinement of synchronous action systems is mainly based on the theory of trace refinement [3]. A trace, or a sequence of values of the global variables, represents an observable behaviour of a system. An abstract action system $\mathcal{A}$ is said to be (trace) refined by the concrete system $\mathcal{C}$, denoted $\mathcal{A} \sqsubseteq \mathcal{C}$, if the traces of $\mathcal{A}$ and $\mathcal{C}$ are equivalent. Notice that, in a trace, several successive equivalent states are considered as a single one.

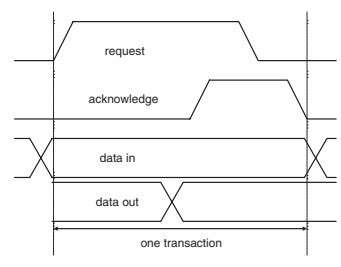

Fig. 1. The four phase handshake

The design of synchronous action systems [10] is based on correctly deriving synchronous representations from asynchronous action systems. An asynchronous system interacts with its environment via an asynchronous communication (handshake) channel, which is composed of two boolean variables [6], denoted req and ack (request, acknowledge), and data variables. The handshake variable $r e q$ is updated by the master system which is the active party of communication requesting tasks from the server system which is the passive party of communication. The variable ack is updated by the server whenever it has completed a task requested by the master. In our approach, the synchronous system is the server component of an asynchronous description and a system 
that models the environment acts as the master. We build our design flow on the four-phase signalling protocol [4] (Fig. 1).

\section{Pipelined Synchronous Action Systems}

In order to obtain a pipelined version of an initially non-pipelined system, we have to introduce / eliminate local variables of the specific system. We do this according to the methods presented in [11], accommodating for our purposes the rules presented in [2]. We continue with the introduction of the pipelining procedure. For performing the translation from an asynchronous to a synchronous representation, the system under analysis has to comply with a set of synchronization requirements [10]. Thus, one of the requirements specifies that it is possible to transform a sequence (for instance $A_{1} ; \ldots ; A_{n}$ ) into a simultaneous assignment $\left(A_{1} \star \ldots \star A_{n}\right)$, and from here, into a synchronous composition, if actions in the sequence do not read what other actions, positioned earlier in the sequence, have just updated. The process preserves the existence of the communication variables $r e q$ and $a c k$ in the synchronous description.

Pipelining Procedure. Let us consider now a synchronous action system, $\mathcal{A}$, obtained from an initial asynchronous system. Thus, $\mathcal{A}$ contains the action $A C K$ that updates the acknowledge signal ack :

$$
\begin{aligned}
& \text { sys } \mathcal{A}(\text { req, ack }: \text { bool; } x, y, a, b: \text { data }):: \\
& \quad \text { |[ var } l ; \text { init } l, x, \ldots, b:=l_{0}, x_{0}, \ldots, b_{0} ; r e q, a c k:=\text { false } \\
& \quad \text { do } A \nabla B \nabla A C K \text { od } \\
& \quad] \mid, \\
& A \hat{=} a:=a^{\prime} \cdot Q_{A}, \quad Q_{A} \widehat{=}\left(r e q \Rightarrow Q_{a}\right) \wedge\left(\neg r e q \Rightarrow a^{\prime}=a\right), \\
& B \hat{=} b:=b^{\prime} \cdot Q_{B}, \quad Q_{B} \widehat{=}\left(r e q \Rightarrow Q_{b}\right) \wedge\left(\neg r e q \Rightarrow b^{\prime}=b\right), \\
& A C K \widehat{=} a c k:=a c k^{\prime} \cdot Q_{K}, Q_{K} \widehat{=}\left(r e q \Rightarrow a c k^{\prime}=t r u e\right) \wedge\left(\neg r e q \Rightarrow a c k^{\prime}=\text { false }\right)
\end{aligned}
$$

Let us now assume that the direct circuit implementation of the actions $A$ and $B$ of the system $\mathcal{A}$ is estimated to be so complex that it cannot be operated with the specified clock frequency. Then the computations of $A$ and $B$ have to be divided into $n(n>1)$ phases each of which is simple enough to be executed in one clock cycle. A set of $n-1$ new local variables is introduced for every action we split, in order to store the results of each phase. The combinational logic that updates the variable ack is a very simple construct. Consequently, the update is possible to be completed in a single clock step. Therefore, the update on the variable $a c k$ is only delayed but not split over $n$ stages.

Pre-analysis Step. Intuitively, the first step in dividing the action $A$ into $n$ pipelined actions is to transform it locally into the atomic $n$-element sequence:

$$
\begin{aligned}
A & \leq A^{\prime}, A^{\prime} \widehat{=} A_{0} ; \ldots ; A_{n-1}, \\
A_{j} & \widehat{=} a_{j}:=a_{j}^{\prime} \cdot Q_{a}^{j}, j=0, \ldots, n-1, a_{n-1}=a
\end{aligned}
$$

The above refinement requires that

$$
\left(Q_{a}^{0} \wedge Q_{a}^{1}\left[a_{0}^{\prime} / a_{0}\right] \wedge \cdots \wedge Q_{a}^{n-1}\left[a_{n-2}^{\prime} / a_{n-2}\right]\right) \equiv Q_{A}
$$


As each action of the sequence $A^{\prime}$ reads the values of the variables updated by the previous action in the sequence, it is not possible to obtain a synchronous

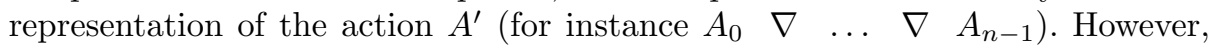
this study is necessary as it indicates what the relations $Q_{a}^{j}, j=0 \ldots n-1$ are (in other words, it shows how to split the execution of action $A$ ). They will be used in the following steps, when we describe the transformation of the system $\mathcal{A}$ into an equivalent pipelined system. Observe that a similar procedure is to be applied to action $B$, too.

Step 1. Buffering. We start the transformation of the action system (1) by introducing $3 \cdot(n-1)$ variables. Consequently, we also have $3 \cdot(n-1)$ new actions. Initially we only modify the original actions so that they have as input the new local variables, instead of the initial global variables:

$$
\begin{aligned}
& \text { sys } \mathcal{A}^{1}(\text { req, ack : bool; } x, y, a, b: \text { data }):: \\
& \text { I[ } \operatorname{var} l, a_{0}, \ldots, a_{n-2}, b_{0}, \ldots, b_{n-2}, r e q_{0}, \ldots, r e q_{n-2} \\
& \text { init } l, x, \ldots, b:=l_{0}, x_{0}, \ldots, b_{0} ; r e q, a c k, r e q_{0}, \ldots, r e q_{n-2}:=\text { false; } \\
& a_{0}, b_{0}, \ldots, a_{n-2}, b_{n-2}:=a_{0_{0}}, b_{0_{0}}, \ldots, a_{n-2_{0}}, b_{n-2_{0}} \\
& \text { do } A^{\prime} \nabla A_{0} \ldots \nabla A_{n-2} \nabla B^{\prime} \nabla B_{0} \ldots \nabla B_{n-2} \\
& \nabla A C K^{\prime} \nabla A C K_{0} \ldots \nabla A C K_{n-2} \text { od } \\
& \text { ]|, }
\end{aligned}
$$

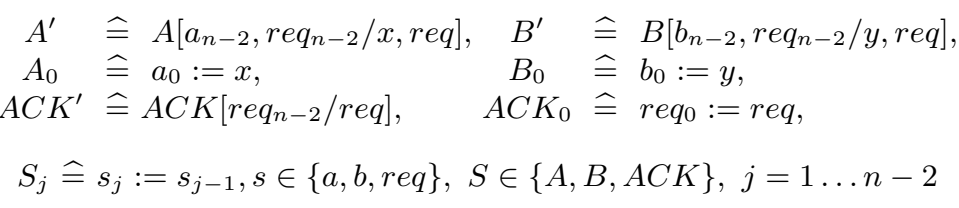

The hardware implementation of the system $\mathcal{A}^{1}$ is shown in Fig. 2.

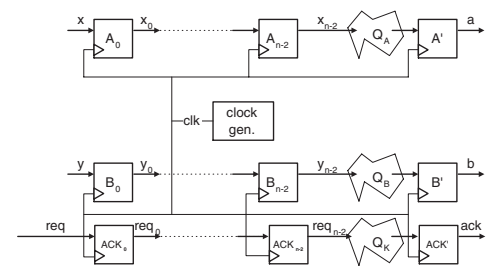

Fig. 2. Intermediate stage in the pipelining process

The result of Step 1 is the refinement $\mathcal{A} \sqsubseteq \mathcal{A}^{1}$. Observe that until requn-2 $=$ true, the system $\mathcal{A}^{1}$ does not modify its observable state (it stutters). Also, after req becomes false, until $r e q_{n-2}=$ false, $\mathcal{A}^{1}$ stutters again.

Step 2. Auxiliary Actions. Next, we introduce $2 \cdot n$ additional actions $D_{0}, \ldots$, $D_{n-1}$ and $C_{0}, \ldots, C_{n-1}$, that synchronously update the new local variables $d_{0}, \ldots, d_{n-1}$ and $c_{0}, \ldots, c_{n-1}$, respectively. We have

$$
\begin{aligned}
& D_{0} \widehat{=} d_{0}:=d_{0}^{\prime} \cdot Q_{D}^{0}, Q_{D}^{0} \widehat{=} Q_{a}^{0}\left[d_{0}^{\prime} / a_{0}^{\prime}\right] \\
& C_{0} \widehat{=} c_{0}:=c_{0}^{\prime} \cdot Q_{C}^{0}, Q_{C}^{0} \widehat{=} Q_{b}^{0}\left[c_{0}^{\prime} / b_{0}^{\prime}\right]
\end{aligned}
$$


For $j=0 \ldots n-1$, the actions $D_{j}$ and $C_{j}$ update the corresponding output variables as specified by the predicates selected in the pre-processing step:

$$
\begin{aligned}
& D_{j} \widehat{=} d_{j}:=d_{j}^{\prime} \cdot Q_{D}^{j}, Q_{D}^{j} \widehat{\widehat{=}}\left(r e q_{j-1} \Rightarrow Q_{a}^{j}\left[d_{j}^{\prime} / a_{j}^{\prime}\right]\right) \wedge\left(\neg r e q_{j-1} \Rightarrow d_{j}^{\prime}=d_{j}\right), \\
& C_{j} \widehat{=} c_{j}:=c_{j}^{\prime} \cdot Q_{C}^{j}, Q_{C}^{j} \widehat{=}\left(r e q_{j-1} \Rightarrow Q_{b}^{j}\left[c_{j}^{\prime} / b_{j}^{\prime}\right]\right) \wedge\left(\neg r e q_{j-1} \Rightarrow c_{j}^{\prime}=c_{j}\right)
\end{aligned}
$$

At the system level we have sys $\mathcal{A}^{1} \sqsubseteq$ sys $\mathcal{A}^{2}$, where $\mathcal{A}^{2}$ is described as

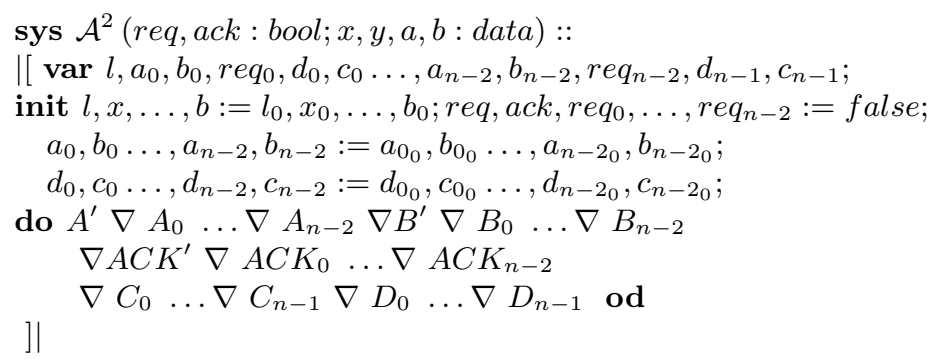

Step 3. Removal of Auxiliary Variables. The Final Description. Next, notice that

$$
\begin{aligned}
r e q \wedge r e q_{n-2} \Rightarrow & \left(Q_{D}^{n-1}\left[a / d_{n-1}\right] \equiv Q_{A}\left[a_{n-2}, r e q_{n-2} / x, r e q\right]\right) \\
& \wedge\left(Q_{C}^{n-1}\left[b / b_{n-1}\right] \equiv Q_{B}\left[b_{n-2}, r e q_{n-2} / y, r e q\right]\right)
\end{aligned}
$$

The interpretation of the above relation is as follows. The update on variable $a$, either according to $Q_{A}$, or to $Q_{D}^{n-1}$, leads to the same result. Therefore, we can write

$$
\begin{aligned}
& A^{\prime} \leq A^{\prime \prime}, A^{\prime \prime} \widehat{=} D_{n-1}\left[a / d_{n-1}\right], \\
& B^{\prime} \leq B^{\prime \prime}, B^{\prime \prime} \widehat{=} C_{n-1}\left[b / c_{n-1}\right]
\end{aligned}
$$

Now we can safely eliminate the variables $a_{n-2}, b_{n-2}$ from the system description, together with the actions that update them. We repeat this procedure for the variables $a_{j}, b_{j}(j=n-3 \ldots 0)$ and for the corresponding actions. Eventually we come to the system $\left(\mathcal{A}^{2} \sqsubseteq \mathcal{A}^{3}\right)$ :

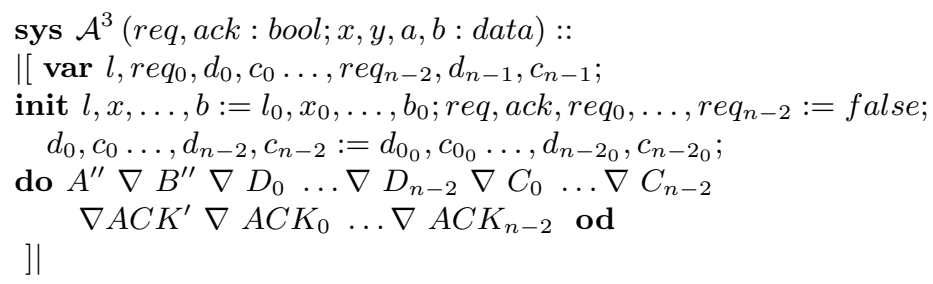

The implementation of the system $\mathcal{A}^{3}$ is shown in Fig. 3.

\section{Conclusions}

We introduced an action systems-based method to transform a non-pipelined synchronous system description into a pipelined structure within a correctness preserving formal framework. The pipelining procedure was viewed as a four-step refinement which started from atomic sequencing of an action and was completed by splitting the action in question into separate synchronous components each 


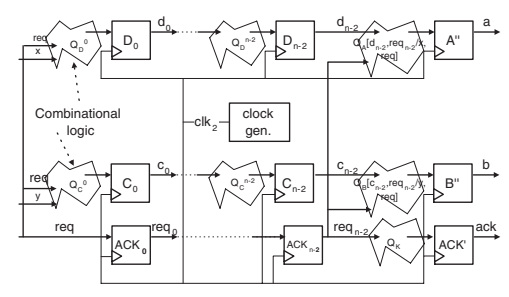

Fig. 3. The system $\mathcal{A}^{3}$ in hardware

of which constituted a stage of the created pipeline. The work presented in this paper was motivated by importance and generality of pipelining in contemporary VLSI design and by the view that the need for formal design methods is becoming more and more obvious with increasing complexity of digital VLSI systems.

Several issues are still to be addressed in further work studies, such as the elimination of the communication variables and its implication in pipelinedsystem modelling, as well as the subject of pipeline control.

\section{References}

1. R. J. R. Back and K. Sere. Stepwise refinement of action systems. Structured Programming, 12:17-30,1991.

2. R. J. R. Back and J. von Wright. Refinement calculus: A Systematic Introduction. Springer. April 1998.

3. R. J. R. Back and J. von Wright. Trace refinement of action systems. In B. Jonsson and J. Parrow, editors, CONCUR'94: Concurrency Theory, Sweden, 1994, volume 836 of LNCS. Springer, 1994.

4. A. Davis and S. M. Nowick. Asynchronous circuit design: motivation, background and methods. Asynchronous Digital Circuit Design, G. Birtwistle and A. Davis (eds.), pages 1-49. Springer, 1995.

5. E. W. Dijkstra. A Discipline of Programming. Prentice-Hall International, 1976.

6. J. C. Ebergen, J. Segers, I. Benko. Parallel Program and Asynchronous Circuit Design. Asynchronous Digital Circuit Design, G. Birtwistle and A. Davis (eds.), Springer, 1995.

7. G. De Micheli. Synthesis and Optimisation of Digital Circuits. McGraw-Hill International Editions, 1994.

8. J. Plosila. Self-Timed Circuit Design - The Action Systems Approach. Ph.D. Thesis, University of Turku, Dept of Applied Physics, Turku, Finland, 1999.

9. J. Plosila and T. Seceleanu. Modeling Synchronous Action Systems. In Proc. of the $17^{\text {th }}$ NORCHIP Conference, Oslo, Norway, pages 242-248. November 1999.

10. J. Plosila, T. Seceleanu. Design of Synchronous Action Systems. In Proceedings of The $13^{\text {th }}$ International Conference on VLSI Design, January 2000, pages 578-583.

11. T. Seceleanu, J. Plosila. Synchronous Pipeline Design in Action Systems. Turku Centre for Computer Science Technical Report No. 403, 2001.

12. J. M. Rabaey. Digital Integrated Circuits - A Design Perspective. Prentice-Hall International, 1996. 